\title{
Rationale for the use of DC microgrids: feasibility, efficiency and protection analysis
}

\author{
M. Seyedmahmoudian, H. Arrisoy, I. Kavalchuk, \\ A. Maung Oo \& A. Stojcevski \\ School of Engineering, Faculty of Science, \\ Engineering \& Built Environment, Deakin University, Australia
}

\begin{abstract}
Electrical power systems are undergoing highly significant changes in their structures. The emergence of renewable energy units in the power generation sector, the use of high-voltage DC in the power transmission sector, and the prevalence of islanded or integrated microgrids in the distribution sector are the strongest evidence supporting this claim. These changes are mostly the consequences of the increasing energy demand rate, climate change, and environmental challenges, as well as the high investment and maintenance cost of the previous structures. Considering these new conditions and according to the recent development in DC/DC conversion topologies and control techniques, different studies have been conducted on how and why DC microgrids outperform AC microgrids. This study discusses the feasibility of the DC microgrid system according to recent developments in power systems. The efficiency and power loss reduction in DC distribution systems are then analyzed, some of the common strategies and devices for protection systems in such networks are reviewed, and the possible and existing challenges in developing the DC microgrids are highlighted. The mathematical calculations and theories for this evaluation are presented to determine the reliable justification for selecting the appropriate microgrid systems.

Keywords: DC microgrids, distributed generation, DC system efficiency, renewable energy systems.
\end{abstract}




\section{Introduction}

Electrical power systems have recently undergone a significant change in principle. The increasing rate of energy demand, global warming, environmental challenges, as well as the dramatic change in the consumption behavior of users, are among the main challenges for planners in the power generation and distribution sectors. As a result, the utilization of renewable energy resources, such as wind and photovoltaic systems, is among the most popular topics in engineering and scientific institutions. The performance of different microgrid systems is one of the fast-developing areas related to the significant change in the power generation and distribution sectors. Factors, such as high investment costs for constructing a large power plant and long transmission lines, low efficiency because of high power loss, environment-related challenges, as well as the high maintenance cost and aging of conventional systems, are some of the main reasons that forced the current power system toward the distributed generation and microgrid approaches.

Microgrids are generally defined as small-scale power systems that include different components, such as distribution generation (DG) units, loads, storage systems, protection, and power electronic device converters associated with controllers, sensors, as well as active and passive components. Microgrids will affect the structure of conventional energy systems where energy is generated by large power plants and is distributed through the transmission lines and central dispatching centers. One advantage of the microgrid is the flexibility to be operated in the AC or DC principles without interfering with other parts of the power system.

The selection of the AC or DC microgrid systems is a critical task because this decision affects different parameters of the power system characteristics, such as power quality, reliability, and stability of the system. This selection also largely alters the investment cost, overall efficiency, and security index of the microgrid system. The AC electrification has dominated the DC power system over the last century and has been a wise choice to a large extent. AC generated power from rotating generators in fossil fueled energy power plants, feasible power protection solutions as a result of zero current intersection, and easy to step up the voltage through the transformers to reduce power loss are the main reasons for the preference of the AC system over the DC system. According to the increasing number of DC-powered loads, as well as the huge utilization of DC-generating renewable energy resources and storage systems, rethinking the selection of AC power in microgrid systems seems logical [1].

This study aims to present a thorough investigation and evaluation of the DC microgrid system. First, this study discusses the feasibility and compatibility of DC electrification in the microgrid system on the basis of current developments. Second, this work provides an analytical study on the effects of the DC system on efficiency improvement and power loss reduction in different sections of the microgrid. This work investigates the common protection topologies developed for DC distribution systems in response to the common concern about the deficiency of the protection system in DC circuits. Finally, the challenges and 
problems of the DC microgrid system are highlighted. In all steps of this study, evaluations are based on electrical engineering fundamentals and are referred to the accredited literature. Initially, a brief overview of the main factors and reasons for the domination of the AC over the DC power system in electrical energy networks is presented.

\section{AC versus DC}

The common use of AC systems in almost all residential and commercial buildings is an undeniable fact. However, this decision was not made abruptly. In the late $18^{\text {th }}$ century, Thomas Edison, the director of Edison Illuminating Company, developed a small power plant in Pearl Street Station in Manhattan to supply customers with low-voltage DC powered lamps. The Edison DC power plant was distributed in three lines of $+110,0$, and -110 volts. The voltage drop across the power lines was significantly high and therefore, transmitting the power to remote areas and other residential neighborhoods far from the main power plant was not a viable option. In 1886, the Gnaz Work Company electrified the large European metropolis of Rome. Meanwhile, George Westinghouse used the newly invented transformer to construct the initial AC system of Westinghouse Electric Company in Pennsylvania, North America. The "Battle of the Currents" involved American and European companies whose heavy investments in one current type or the other led them to hope "their" current type would be favored. Transformers provided an inexpensive method to increase the voltage of AC from several thousand volts and back down to usable levels. At high voltages, the same power could be transmitted at a lower current, which resulted in less power loss attributed to wire resistance. As a result, large power plants could be located many miles away and may service a large number of customers [2-4].

This controversy intensified in 1887 when Nicola Tesla registered several patents in the United States. These patents were related to the AC distribution system, which Westinghouse Electric and Manufacturing Company supported. The patents comprised all components of the AC distribution system which include polyphase AC motors, power transmissions, generators, transformers, and lighting. The findings of Tesla significantly influenced the superiority of the AC system in the "Battle of the Currents." In 1893, Westinghouse won a contract to build a hydroelectric dam to harness the power of Niagara Falls and transmit AC to Buffalo, New York. This project was completed on November 16, 1896, and AC power began to power industries in Buffalo. This milestone marked the decline of DC in the United States and provided a successful experience for future worldwide power distribution and transmission projects [5, 6].

Edison exerted considerable effort to increase the efficiency of the DC system and reduce line loss along with René Thury who used a series of motor-generators to create a high-voltage DC (HVDC) system in the 1880s. Such system could be used to transmit DC power over long distances. However, HVDC was never adopted for almost a century because of the high cost and maintenance that it required. Despite considerable efforts from Edison and his fellows to discourage the selection of AC electrification, the use of the transformer significantly helped 
AC superiority at that time. Transformers facilitate the voltage increase and reduce the line losses for transmission to long-distance areas while stepping down the voltage at the destinations. However, the popularity of the AC systems in that period of time can be attributed to the limited knowledge of semiconductor materials and power electronic devices. With the invention of semiconductor electronics in the 1970s, economically transforming between AC and DC became possible. $[5,7,8]$.

\section{Feasibility of DC microgrid system}

The transition from AC to DC is not simple to any extent and has major effects on the current electrical system structure. In this section, the feasibility of this transition and the DC microgrid system will be discussed on the basis of recent developments in electrical power systems.

There are many factors to take into consideration in order to make an accurate assessment of the feasibility of DC microgrids over AC systems. Some of the technical challenges are a simple application of known and existing technical solutions to implement DC microgrids, however there are some issues that require a concerted effort from the energy generator and distribution companies all the way to appliance manufacturers and users. To date the lack of international DC power transmission and distribution network standards have made it very difficult to implement DC systems. However the continued growth of distributed energy generation, the take up of renewable energy generation systems by the public throughout the whole world, the importance invested in carbon reduction and energy efficient systems due to energy price increases has made the feasibility of introducing DC microgrids that much more likely. When considering implementation of microgrids AC or DC several factors need to be taken into consideration. These factors may include but are not limited to:

- The type of energy generation systems available

- The size of the microgrid

- The requirements of the users / customers

- The capital investment in the technology

- The return in investment

Once these impeding factors are considered and resolved the technical considerations will need to be investigated. The size of the microgrid and the types of available distributed generation sources can be used to determine the technical specifications for an implementation of a DC microgrid. As we have discussed one of the current technical limitations are the limited number of appliances that run on a DC bus. However before appliance manufacturers can produce these appliances, some standards will need to be adopted. These standards will need to provide the detailed technical specifications for such things as line voltages to enable manufacturers to have something to work with. As the price of renewable energy generation falls while the overall cost of energy rises the feasibility of DC microgrids that produce clean energy and efficient power is assured. 


\subsection{Renewable energy generation units}

As a result of the tremendous development in the utilization of renewable energy resources and the growing concerns on climate change, most microgrid systems have been integrated with the use of distributed renewable energy generation systems, such as photovoltaic (PV) and wind power generation units. The output of a photovoltaic system has a DC form, which is delivered and controlled through DC/DC converters and the associated maximum power point tracking (MPPT) controllers. In a wind power system, the generated power is initially AC. However, wind turbines are incapable of directly controlling frequency and voltage while producing electricity because of variable wind speed. Regardless of whether an $\mathrm{AC}$ or DC system is used, the rectification step is required before the wind turbine is connected to the main grid. Therefore, assuming that renewable energy systems are the main generation units in the microgrid, the DC system is apparently more feasible than the AC microgrid in the generation part of the system. This result can mainly be attributed to the fact that one step of the conversion process was removed in the integration of the generation units into the grid.

\subsection{Storage system}

The generation characteristic of renewable energy resources is not normally compatible with the consumption behavior of users. For instance, the generation of a residential building that is integrated with a PV system is at the maximum level during the day, whereas the highest energy consumption level is during the evening. Therefore, storing the generated energy during the off-peak period and consuming this energy during the peak period with the use of the appropriate energy storage systems are necessary for microgrids, especially in the islanded mode. Energy storage devices produce or operate internally in DC and thus utilize DC/DC converters and DC/AC converters to connect to the AC distribution system. However, this DC-to-AC conversion step can be avoided in DC microgrids $[9,10]$.

\subsection{DC appliances}

As the result of the advances in power electronics, the number of DC loads has dramatically increased. Appliances that implement electronics typically have a switched-mode power supply and converters between the grid and its internal components. To this end, these appliances typically have AC/DC and DC/DC stages to connect to the grid. Efficiency is improved by removing one of the conversion stages, which is discussed in the following steps [9].

\subsection{Emergence of electric vehicles}

The emergence of electric vehicles will significantly affect all aspects of technology, and distribution and electrification systems are no exceptions. Most plug-in hybrid electric vehicles implement AC/DC converters to charge and 
discharge their batteries when connected to the grid. Therefore, charging the storage units in the DC-powered distribution system is more feasible when the appropriate standards are defined [9].

\section{Power loss reduction in the distribution line}

Efficiency of the DC microgrid system versus the AC microgrid system

Considering that all factors are used to compare the AC and DC microgrid systems, efficiency is one of the strongest criteria through which the priority of these systems can be identified. The power loss between the generation units toward the loads should generally be minimized to maximize microgrid system efficiency. Fundamentally, the amount of power loss in each unit is affected whether a DC system or an AC system is used. In this section, the effect of an AC or DC system on the efficiency of main microgrid parts, such as the distribution lines, storage systems, conversion steps, and loads are analyzed.

The lack of any reactive power and its relevant issues in the distribution system are some of the advantages of the DC system. As regards efficiency, the power loss in the DC microgrid is only related to the active power loss attributed to the resistive loads in the system. Moreover, no skin and proximity effects in the zero frequency DC distribution systems are found except for the removal of the reactive power loss. This results in further power loss reduction with the ohmic resistance reduction. The power loss reduction discussed in Shenai and Shah [10] and Asad and Kazemi [11] is expressed as follows:

$$
P l_{-} R_{\text {lineDC }}=P l_{\text {lineAC }}\left[1-\left(\frac{P_{D C}}{P_{A C}}\right)^{2} \frac{(\cos \Phi)^{2}}{S}\right]
$$

where $P_{D C}$ and $P_{A C}$ represent the power consumption in the DC and AC systems, respectively; $S$ is the skin and proximity effect coefficient; and $\cos \Phi$ is the power factor of the AC system.

\subsection{Power loss reduction in the DC storage system}

The storage system operation integrated to the microgrid is the DC power. Therefore, all components associated with the storage system operate with the DC power. Applying these storage systems in the AC microgrid or distribution system requires additional conversions for the charging and discharging modes. The rectification step is apparently needed at the input. Moreover, AC conversion is required at the system output. Hence, using this system in the DC microgrid results in a significant overall power loss reduction expressed as follows [11] 


$$
P l_{-} R_{S D C}=P_{\text {inDC }}\left(1-\eta_{\text {rec }}\right)+P_{\text {outAC }}\left(\frac{1}{\eta_{\text {inv }}}-1\right)
$$

where $\eta_{\text {rec }}$ and $\eta_{\text {inv }}$ are the rectifier and inverter efficiencies, respectively; and $P_{\text {in }}$ and $P_{\text {out }}$ are the input and output active power of the system, respectively. The total power loss reduction of the DC storage system is expressed as follows if $\eta_{S D C}$ is considered as the DC storage system efficiency [11]:

$$
P l_{-} R_{S D C}=P_{i n D C}\left[\left(1-\eta_{\text {rec }}\right)+\eta_{S D C}\left(\frac{1}{\eta_{i n v}}-1\right)\right]
$$

\subsection{Power loss reduction in the conversion units}

\subsubsection{Power electronic losses}

One of the main sources of power loss in the AC microgrid is in the AC/DC conversion process, which supplies the required voltage either for the DC loads or the uninterruptible power supply in office facilities or variable speed drives for heating, ventilation, and air conditioning devices. Moreover, most of the DG units in the microgrid generate the DC power. Therefore, several numbers of additional conversion units are imposed to the system in AC microgrids, whereas these conversion units are avoided in DC microgrids consequent to the energy savings in the overall microgrid system [9]. The main components in the rectifiers, $\mathrm{DC} / \mathrm{DC}$, and DC/AC converters are the power electronic devices. Therefore, the main power loss source in the conversion step is related to the loss of the power electronic components.

\subsubsection{Conduction losses}

The conducting losses are related to the losses spent in the resistances, which are associated with all power electronic devices (e.g., insulated-gate bipolar transistors (IGBTs), metal-oxide-semiconductor field-effect transistors, capacitors, and diodes). Assuming the resistive part of these components as the $R_{\text {loss }}$, the ohmic loss when these components are in operation is described as follows:

$$
P l_{\text {Ohm }}=R_{\text {loss }} I^{2}
$$

\subsubsection{Switching losses in power switches}

The use of switches is hardly ideal in practice. First, switches are not capable of immediately turning on and off in one command. The voltage across the switch and the current through it hardly become zero in reality when switching occurs. These two components overlap in a fraction of a second, which causes power loss indicated by eqn. (5) to (8) and fig. 1. 
76 Energy and Sustainability V: Special Contributions

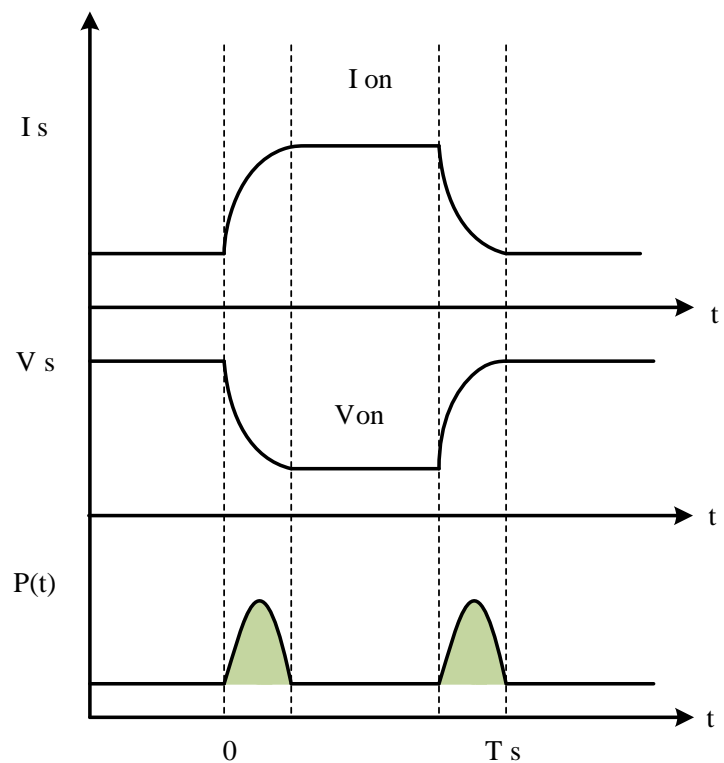

Figure 1: Current through the switch, voltage across the switch, and instantaneous power loss.

$$
\begin{aligned}
& I_{I G B T}=\left\{\begin{array}{cc}
\frac{t}{t_{\text {on }}}\left(I_{\text {on }}-I_{\text {off }}\right)+I_{\text {off }} & 0 \leq t \leq t_{\text {on }} \\
I_{\text {on }} & t_{\text {on }} \leq t \leq T_{s}-T_{\text {off }} \\
-\frac{t-T_{s}}{t_{\text {off }}}\left(I_{\text {on }}-I_{\text {off }}\right)+I_{\text {off }} & T_{s}-T_{\text {off }} \leq t \leq T_{s}
\end{array}\right. \\
& V_{\text {IGBT }}=\left\{\begin{array}{cc}
-\frac{V_{\text {off }}-V_{\text {on }}}{t_{\text {on }}}\left(t-t_{\text {on }}\right)+V_{\text {on }} & 0 \leq t \leq t_{\text {on }} \\
V_{\text {on }} & t_{\text {on }} \leq t \leq T_{s}-t_{\text {off }} \\
\frac{V_{\text {off }}-V_{\text {on }}}{t_{\text {off }}}\left(t-\left(T_{s}-t_{\text {off }}\right)\right)+V_{\text {on }} & T_{s}-t_{\text {off }} \leq t \leq T_{\text {s }}
\end{array}\right. \\
& P_{\text {inst }}=V_{I G B T} \times I_{I G B T} \\
& P_{\text {ave }}=\frac{1}{T} \int_{0}^{T} P_{\text {inst }} d t=\frac{V_{\text {off }} I_{\text {on }}}{T_{\text {s }}}\left(\frac{t_{\text {on }}+t_{\text {off }}}{6}\right)+\frac{V_{\text {on }} I_{\text {on }}}{T_{\text {s }}}\left(T_{s}-t_{\text {off }}-t_{\text {on }}\right)
\end{aligned}
$$


where $P_{\text {inst }}$ is the power at an instant; and $P_{\text {ave }}$ is the total power loss, which includes the switching loss in the first part of eqn (8) and the conduction loss in the second part of eqn (8). $t_{\text {off }}$ and $t_{\text {on }}$ represent the turn-off and turn-on times, respectively, while $V_{\text {on }}$ and $I_{\text {off }}$ are the conduction voltage and the leakage current, respectively. The inverter model is used to simulate such power losses for the chosen pulse-width modulation schemes while the values for the modulation index, switching frequency, DC-link voltage, and load are adjusted.

\subsubsection{Diode forward and recovery losses}

The power loss across the diode is evaluated under different modes as forward and recovery losses. This evaluation is given as follows:

$$
P d l_{t}=\text { Pld }_{\text {on }}+\text { Pld }_{\text {off }}
$$

In addition to the power loss caused by the voltage drop equal to $0.7 \mathrm{~V}$ across the diode, a conducting loss caused by the associated resistance occurs during the forward bias $[9,12]$. The total power loss during the forward bias is calculated as follows:

$$
P l d_{o n}=V d_{o n} \times I d_{a v e}+r_{\text {Ton }} \times I d_{r m s}^{2}
$$

As shown in fig. 2, power loss occurs during the reverse bias because of the reverse current flowing through the diode for a period of $t_{r r}$. This power loss is calculated as follows:

$$
\text { Pld }_{\text {off }}=0.5 I_{R R M} V_{o} \times t_{r r} \times f_{s}
$$

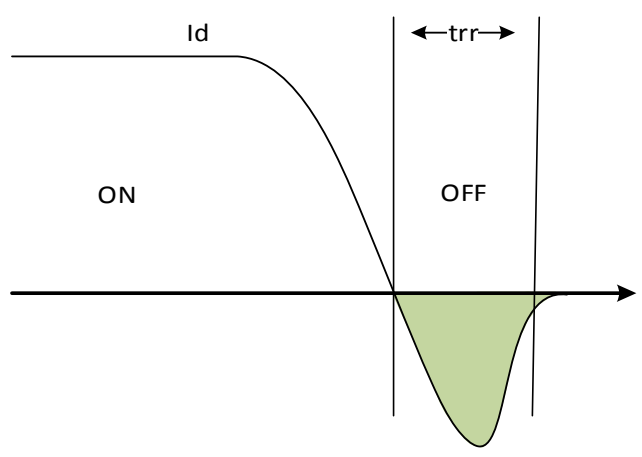

Figure 2: Diode recovery loss.

The preceding losses reduce the efficiency of any power electronic device in the circuit. Therefore, reducing the conversion steps in the circuit reduces power loss in the overall system. Numerous studies have been conducted as regards the efficiency of different rectifiers and inverters used in electrical systems $[1,13]$. Most of the studies indicate that the efficiency of different converter topologies is $95 \%$ or higher. Assuming the efficiency ratio of $94 \%$ and $97 \%$ for DC-DC and 
DC-AC converters, respectively, fig. 6 illustrates the loss comparison between the $\mathrm{AC}$ and the DC microgrids according to the number of converters associated in the system [1].
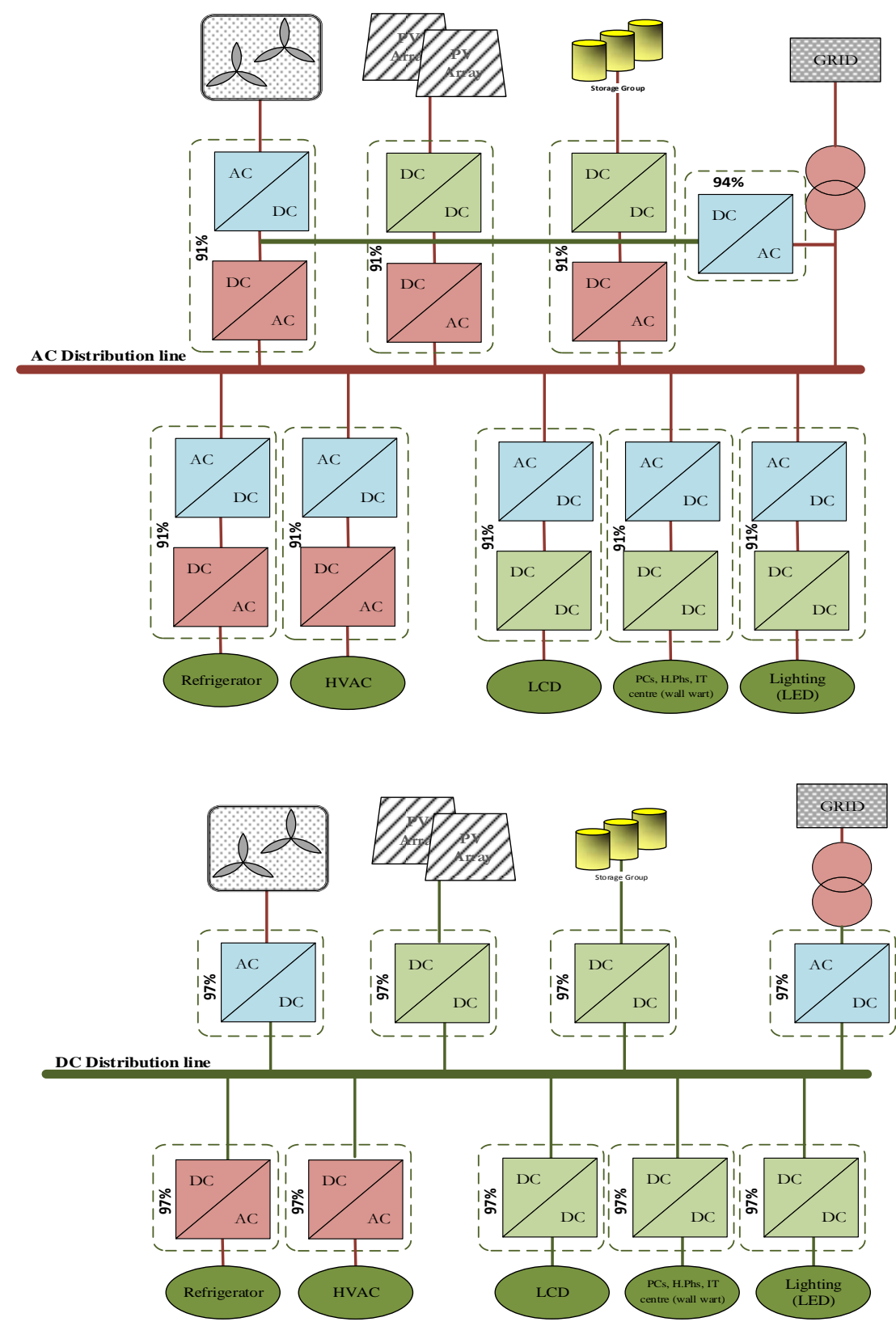

Figure 3: Composition of AC and DC microgrid systems. 


\subsection{Energy efficiency by appliances}

Using the DC distribution and microgrid systems becomes more common because of the expansion of the microgrid based on renewable energy. This improvement might result to the new standardization in home and commercial electrification, which can even revolute the manufacturing specifications of most daily used appliances. Garbesi $[13,14]$ investigated the potential energy efficiency gain if household appliances are used in DC systems. Their study aimed to gain insight on the impact of modifying the appliances used in households and industries. They estimated that an additional 33\% (weighted average) can be achieved. Replacing incandescent light bulbs for LED lights is about 70\% more efficient. As regards heating, replacing electrical resistances for heat pumps operated with brushless DC permanent magnet motors (BDCPM) saves up to 50\%. Replacing induction motors, compressors, or pumps for the BDCPM in cooling equipment reduces energy consumption by $5 \%$ to $50 \%$. Fig. 3 shows the possible energy savings in switching from the standard technologies to the most efficient and more DCcompatible technologies [9].

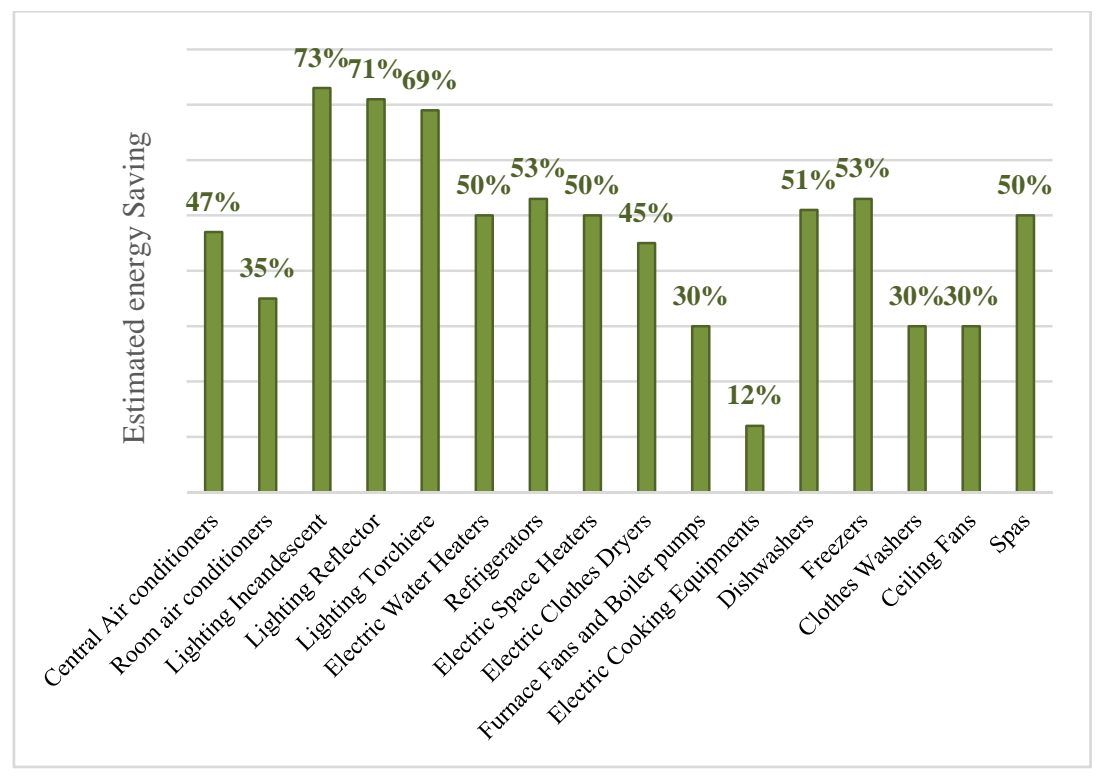

Figure 4: Energy savings possible from switching from standard technologies to the most efficient DC-internal technologies.

\section{Protection systems in the DC microgrid}

The protection system is one of the most challenging parts of the DC microgrid. The main obstacles of the DC protection systems are the difficulty of avoiding the DC arc, very expensive DC protection devices, immaturity of the protection 
strategies, and the lack of standard SFS 6000. Moreover, the fuses and relays do not function well because the short-circuit currents in the power converters are not long enough to allow for these protection systems to react [1]. Accordingly, different studies have designed effective methods to address the preceding problems. This study highlights current techniques and devices presented in Park and Candelaria [15].

Avoiding any protection system in the DC circuit and using protection devices (i.e., circuit breakers and fuses) in the AC side once the fault happens are the simplest and commonly used protection techniques. The main problem associated with these methods is de-energizing the whole DC power system from fault detection to removal. Furthermore, these techniques are not appropriate for a purely DC-powered microgrid, where the DC is used in transmitting power from the AC generation units to the loads $[15,16]$. Another method of protecting the system from excessive fault current is by limiting the bus current under fault conditions. The fault current limiters are used in conjunction with circuit breakers (CBs). The advantages of limiting the fault current include a ride through for temporary faults, safe operation of the switchgear, and low-cost system by switchgear capacity reduction. Several devices (e.g., superconductor saturated inductors and power electronic devices) have been utilized to limit the fault current $[15,17,18]$.

Solid-state switches have been widely used in the protection systems of the DC microgrid owing to the development of semiconductor devices and the limitation of conventional circuit breakers in the DC power system. The gate turn-off thyristor (GTO) block the high voltage in off state and has low voltage drop in on state. However, the deficiency of the GTO appears in high-frequency switching [15].

IGBTs are widely used in low-voltage systems $(<1200 \mathrm{~V})$. Their advantages include fast interruption time and high short-circuit current withstanding capability. However, IGBTs also have a disadvantage (i.e., high conduction loss). Meanwhile, integrated-gate commutated thyristors (IGCTs) have lower conduction losses of a thyristor with the turn-off capability of a transistor. The IGCTs have high voltage and current ratings similar to the IGBTs. Furthermore, the conduction loss is relatively low. The solid-state CB should be bidirectional so the microgrid bus allows power flow in either direction. Fuses or molded case CBs (MCCBs) are utilized in DC systems. Fuses are problematic because they trip the faulted line, thereby leaving an energized unfaulted pole. The drawback of fuses and MCCBs is that neither conducts an autonomous control, albeit this problem solved in MCCBs. Human intervention is required in the event of a fault to re-energize the system once the fault has been removed [15].

\section{Conclusion}

This paper presents a comparative study on the DC and the AC microgrid systems according to recent electrical power system developments. The feasibility of the DC microgrid is discussed first as regards the recent advances in the distributed generation units and power electronics. A mathematical analysis is then presented 
to evaluate efficiency improvement and power loss reduction of the DC electrification in different parts of the microgrid. The common and recent approaches in the protection system of the DC microgrid are covered in subsequent sections. The challenges, uncertainties, and problems associated with the DC electrification in the microgrid systems are also discussed. The analysis shows that the recent changes in the power generation sector and the integrating distributed generation units into the electrical power systems strongly support the DC electrification of the microgrid. Moreover, the lack of reactive power and lower electromagnetic interference in addition to the significant power loss reduction in different parts of the microgrid (e.g., distribution lines, storage system, and power conversion units) firmly prove the higher efficiency of the DC microgrid compared to the AC microgrid. However, further development of DC microgrid systems faces numerous challenges. These problems include the lack of proper standards for voltage levels and protection strategies, more expensive equipment, less adaptability with the current power system, and immaturity of protection strategies.

\section{References}

[1] J. J. Justo, F. Mwasilu, J. Lee, and J.-W. Jung, “AC-microgrids versus DCmicrogrids with distributed energy resources: A review,” Renewable and Sustainable Energy Reviews, vol. 24, pp. 387-405, 2013.

[2] T. McNichol, AC/DC: The savage tale of the first standards war: John Wiley \& Sons, 2011.

[3] M. Josephson, “Edison,” McGraw-Hill Paperbacks, New York: McGrawHill, 1969, vol. 1, 1969.

[4] C. L. Sulzberger, "Triumph of ac-from Pearl Street to Niagara,” Power and Energy Magazine, IEEE, vol. 99, pp. 64-67, 2003.

[5] C. L. Sulzberger, "triumph of ac, part 2."

[6] G. Neidhofer, "Early three-phase power [History]," Power and Energy Magazine, IEEE, vol. 5, pp. 88-100, 2007.

[7] P. Schavemaker and L. Van Der Sluis, Electrical power system essentials: John Wiley \& Sons, 2008.

[8] B. A. Thomas, I. L. Azevedo, and G. Morgan, "Edison Revisited: Should we use DC circuits for lighting in commercial buildings?” Energy Policy, vol. 45, pp. 399-411, 2012.

[9] M. A. V. Evans, "Why Low Voltage Direct Current Grids?” 2013.

[10] K. Shenai and K. Shah, "Smart DC micro-grid for efficient utilization of distributed renewable energy,” in Energytech, 2011 IEEE, 2011, pp. 1-6.

[11] R. Asad and A. Kazemi, "A Quantitative Analysis of Effects of Transition from AC to DC System, on Storage and Distribution Systems," in Power and Energy Engineering Conference (APPEEC), 2012 Asia-Pacific, 2012, pp. 1-5.

[12] R. Bürkel and T. Schneider, "Fast Recovery Epitaxial Diodes (FRED)Characteristics,” Applications, Examples, 1998. 
[13] K. Garbesi, "Optimizing energy savings from direct-DC in US residential buildings,” 2012.

[14] K. Garbesi, “Catalog of DC appliances and power systems,” 2012.

[15] J. D. Park and J. Candelaria, "Fault Detection and Isolation in Low-Voltage DC-Bus Microgrid System,” Power Delivery, IEEE Transactions on, vol. 28, pp. 779-787, 2013.

[16] T. Lianxiang and O. Boon-Teck, "Locating and Isolating DC Faults in Multi-Terminal DC Systems,” Power Delivery, IEEE Transactions on, vol. 22, pp. 1877-1884, 2007.

[17] L. Fang, C. Jian, L. Xinchun, K. Yong, and D. Shanxu, “A novel solid state fault current limiter for DC power distribution network," in Applied Power Electronics Conference and Exposition, 2008. APEC 2008. Twenty-Third Annual IEEE, 2008, pp. 1284-1289.

[18] U. Ghisla, I. Kondratiev, and R. A. Dougal, "Branch circuit protection for DC systems,” in Electric Ship Technologies Symposium (ESTS), 2011 IEEE, 2011, pp. 234-239. 